\title{
Magnetically-Confined Fe-Mn Bimetallic Oxide Encapsulation as an Efficient and Recoverable Adsorbent for Arsenic(III) Removal
}

\author{
Li Yu, Huijuan Liu,* Chunlei Liu, Huachun Lan, and Jiuhui Qu
}

Synthesis of bimetallic-oxide-encapsulated magnetic nanoparticles is still significantly challenging and has rarely been attempted previously, due to the effects of lattice mismatch, weak chemical interactions and variances in growth rates between different components, as well as the difficulty in process control for uniform co-deposition. In the present work, Fe-Mn bimetallic oxide (FMBO) nanoplatelet encapsulated magnetic nanoparticles (Mag$\mathrm{FeMn}$ ) are prepared by controlled engineering of the interparticle coupling of $\mathrm{Fe}_{3} \mathrm{O}_{4}$ and $\mathrm{FMBO}$, with its multifunctional capabilities highlighted in terms of the potentially superior $\mathrm{As}$ (III) sequestration and convenient recoverability. Multiple characterization techniques are employed to examine the derived morphologies and to accurately resolve both compositionally and magnetically the hierarchical structure in detail. The synthesized magnetic composites retain highly porous structure with the main components of $\mathrm{Fe}_{2} \mathrm{O}_{3}$, $\mathrm{FeOOH}, \mathrm{Fe}_{3} \mathrm{O}_{4}$, and $\mathrm{Mn}_{3} \mathrm{O}_{4}$. Mag-FeMn exhibits a quite competitive high capacity for As(III) capture (56.1 $\mathrm{mg} \mathrm{g}^{-1}$ ), whereby As(III) oxidation coupled with synchronous sorption contributes to the improved performance. The unique heterostructure of FMBO encapsulation with an embedded magnetic core would be applicable to help with rational synthesis of other bimetallic oxide encapsulated magnetic nanoparticles, and definitely shows promise for the development of new nanotechnology enabled approaches for adsorptionbased water purification.

\section{Introduction}

Bimetallic oxides have long been explored as promising candidates for adsorbents, heterogeneous catalysts, and pseudocapacitors, by virtue of the special properties derived from their large surface areas, inherent adsorptive affinity, and abundant active sites with superior redox activity and stability. ${ }^{[1]}$ Indeed, suitable heterostructure engineering of different metal oxides

L. Yu, Prof. H. Liu, C. Liu, Dr. H. Lan, Prof. J. Qu

Key Laboratory of Drinking Water

Science and Technology

Research Center for Eco-Environmental Sciences

Chinese Academy of Sciences

Beijing 100085, P. R. China

E-mail: hjliu@rcees.ac.cn

L. Yu, C. Liu

University of Chinese Academy of Sciences

Beijing 100049, P. R. China

DOI: 10.1002/ppsc.201600009 in most cases has been found to endow the resultant composites with integrated functionalities, and even synergistically enhanced physicochemical responses or entirely novel properties could be envisioned beyond the limitations imposed by the individual components. ${ }^{[2]}$ Currently Fe-Mn bimetallic oxide (FMBO) has aroused concerns for its superior efficiency in removal of arsenite $(\mathrm{As}(\mathrm{III})),{ }^{[3]}$ which is much toxic and mobile with higher risk than arsenate $(\mathrm{As}(\mathrm{V}))$, for both the environment and human health. Yet for all that, the inconvenience and inefficiency posed by the separation and recovery of nanoparticles via conventional filtration or centrifugation tend to restrict the sustainability and economics of its application. Colvin's group then employed $\mathrm{Fe}_{3} \mathrm{O}_{4}$ nanocrystals for water purification with facile magnetic separation and recovery, and great success has been achieved for arsenic removal. ${ }^{[4]}$ However, bare magnetite particles are highly sensitive to oxidation upon exposure to atmosphere or other oxidizing environments, ${ }^{[5]}$ and usually have limited sorption capacities of arsenic especially for those with large sizes. ${ }^{[4 a]}$ Multicomponent nanoheterostructure architectures, capable for both pollutant abatement and material recovery effectively, therefore, are pursued to address the issue.

Thanks to recent synthetic achievements, the development of versatile protocols in architectural design and control has advanced the synthetic chemistry research beyond conventional nanoparticles into multifunctional heterostructures. These novel materials can be constructed with tailored topologies and geometric parameters that are independently tunable over a wide size range, under thermodynamically controlled and kinetically driven processes. ${ }^{[2,6]}$ In particular, magnetic nanoparticles (MNPs) have proven to be ideal building blocks for hybrid structure construction, showing innovative prospects as adsorbents, catalytic platforms, and as tools in biomedical research. ${ }^{[7]}$ The high magnetic susceptibility would endow the composites with capabilities for facile separation and guided site-specific delivery, as well as possible application in environmental remediation and biomedical diagnostics and therapeutics. Such magnetic heterostructures are commonly obtained by using silica, carbon, or polymer shells as linkers 


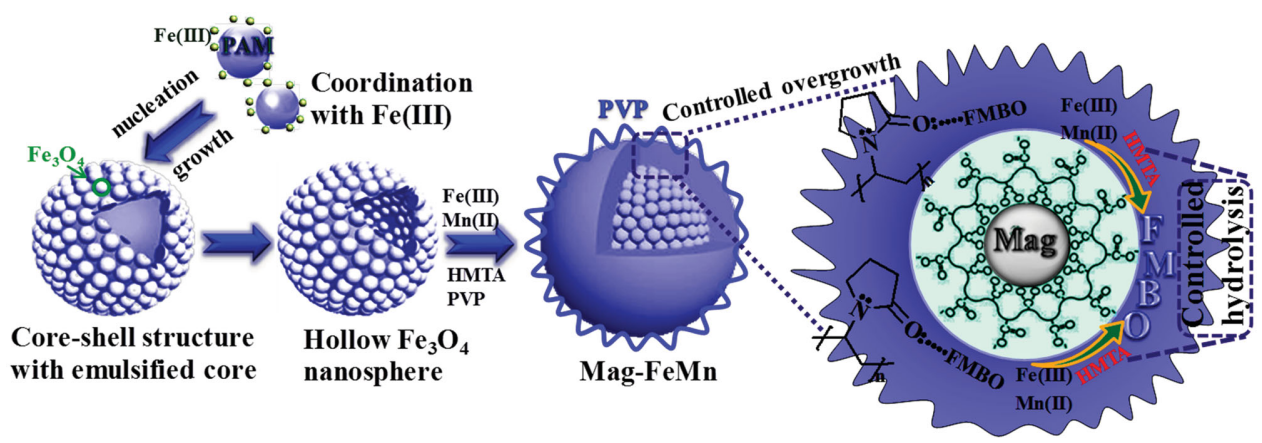

Scheme 1. Schematic representation of the formation of the as-synthesized adsorbents.

for further functionalization, and the protective coatings can also guarantee particle stability. Nevertheless, additional modification and post-treatment are often required in the above synthetic process. ${ }^{[6]}$ A one-pot facile strategy toward modified MNPs with cross-linking groups or layers, as the seeds for subsequent nucleation and growth of other components, therefore, is highly desirable. On the other hand, lattice mismatch and weak chemical interaction of different components may lead to high surface-interface energy between discrete domains, such that ideal core-shell architecture sometimes can be difficult to realize. ${ }^{[8]}$ In addition, metal oxides are prone to aggregation, since the high reactivity of metal precursors always leads to fast homogeneous oxide nucleation. Note also that the nucleation and growth rates between the metal precursors can vary significantly, and uniform co-deposition is often difficult to achieve or control. Such challenges give rise to considerable difficulty in preparation of bimetallic oxide encapsulated magnetic nanoparticles, which has rarely been attempted previously. In fact, rapid homogeneous oxide nucleation and extensive aggregation tend to cause large proportions of bimetallic oxide clusters and dispersions. ${ }^{[9]}$ If controlled deposition cannot be guaranteed, facile magnetic separation and recovery can hardly be achieved. So, controllable architecture of bimetallic oxide encapsulated magnetic nanoparticles, we believe, especially merits further exploration.

Capping agents are usually utilized for tuning the highenergy surface of nanoparticles and stabilizing their aggregation. ${ }^{[10]}$ So far, considerable related achievements have already been attained in hybrid nanocrystal engineering with core-shell structure, ${ }^{[11]}$ hollow structure, ${ }^{[12]}$ and other heterostructures. ${ }^{[13]}$ Specifically, Chen and co-workers ${ }^{[8 c]}$ have recently developed a general strategy for synthesis of a series of different seed@ ZnO and Au@oxide (or metal sulfide) core-shell nanoparticles, based on either seed-ligand-shell or seed-ligandpolyvinylpyrrolidone(PVP)-shell interactions. With a fundamental understanding of the multiple roles of PVP, this methodology can lead the way toward the facile preparation of other hierarchical nanocomposites.

Considering the superior efficiency of FMBO in arsenite (As(III)) removal, ${ }^{[3]}$ in this work, an FMBO encapsulated magnetic nanoparticle (Mag-FeMn) was successfully fabricated by cooperative assembly of $\mathrm{Fe}_{3} \mathrm{O}_{4}$ and FMBO. To assure the controlled deposition of FMBO, a highly porous $\mathrm{Fe}_{3} \mathrm{O}_{4}$ core with excellent stability, dispersibility, and hydrophilicity was first obtained through a one-pot facile approach in aqueous solution.
This could then be used as an ideal seed for further construction of FMBO on the surface owing to the affinity of the functional groups or layers for the metal precursors. PVP was also used to control the nanoparticle aggregation and overgrowth. Although the efficient removal of As(III) has always been particularly problematic in water pollution control and quality restoration, Mag-FeMn forges a new paradigm for this process, whereby simultaneous oxidation and adsorption enable the improved performance, with effective material recovery at the same time.

\section{Results and Discussion}

Hollow $\mathrm{Fe}_{3} \mathrm{O}_{4}$ cores with superior dispersibility, stability, and hydrophilicity were fabricated via a one-pot facile approach in aqueous solution. Hollow structures can be adopted as suitable magnetic cores, because the hollow interiors and permeably porous shells provide more accessible reactive sites for incorporating active components (FMBO) inside the interior compartments or at the surface. ${ }^{[14]}$ Owing to the special properties associated with their high surface areas, low densities and multiphase heterogeneous interfaces, promoted metal-oxide loading, and uniform surface-deposition can be anticipated as a result of the enhanced accessibility of the metal precursors to the interfaces. In the synthesis process (Scheme 1), polyacrylamide (PAM) was considered to be dispersed in the aqueous medium in the form of quasiemulsion microdroplets, which provided nucleation centers for coordination with $\mathrm{Fe}(\mathrm{III})$ and the subsequent growth toward $\mathrm{Fe}_{3} \mathrm{O}_{4}$ nanocrystals. The reducing property associated with citrate at high temperature and alkaline conditions provided by urea facilitated $\mathrm{Fe}_{3} \mathrm{O}_{4}$ formation. Note that the microheterogeneities of the system also ensured an increased viscosity for the reaction medium and thus a controlled rate of nucleation and growth from the supersaturated solution. The continuous hydrothermal reaction and the finally repeated washings led to dissolution or leaching out of the emulsified core, and gave rise to porous hollow nanospheres. It would also be reasonable to expect that sodium polyacrylate was generated through hydrolyzation of PAM. Both sodium polyacrylate and citrate could serve as capping agents to guarantee particle stability and surface functionalization with carboxyl ligands. Note also that hollow microspheres were also favored to develop from the in situ gas bubbles created by urea decomposition. Earlier reports obtained hollow structures or 
other superstructures as well, assisted by intentionally or accidentally created microbubbles. ${ }^{[15]}$ As such, a well-defined hollow $\mathrm{Fe}_{3} \mathrm{O}_{4}$ core was achieved with excellent dispersibility, hydrophilicity, and wettability for abundant carboxyl groups conjugated on the surface, which should show high affinity toward metal precursors and allow for direct surface encapsulation due to the expected low interfacial energy. Owing to the surface-binding hydrophilic ligands, the as-prepared $\mathrm{Fe}_{3} \mathrm{O}_{4}$ nanoparticles showed a high degree of dispersibility in water. The stable suspension could be maintained during static settling for more than $3 \mathrm{~d}$, with limited interparticle agglomeration and fusion (Figure S1a, Supporting Information). The uniform and monodisperse nanoparticles observed from the TEM micrograph further evidenced the superior dispersibility, stability, and hydrophilicity of the magnetite core in aqueous solution (Figure S1b, Supporting Information). These properties were usually crucial for the controlled coating process. Considering the high reactivity of metal precursors and rapid homogeneous oxide nucleation, PVP was introduced to control the aggregation and overgrowth via interactions with iron and manganese ions. Hexamethylenetetramine (HMTA) was used to ensure a slightly alkaline environment and to control the hydrolysis of iron and manganese ions as well. The as-prepared adsorbents were finally obtained according to the above procedure.

The representative scanning electron microscope (SEM) and transmission electron microscope (TEM) images (Figure 1a) clearly manifest typical sphere-like morphologies with diameter of $\approx 200 \mathrm{~nm}$ for the as-prepared $\mathrm{Fe}_{3} \mathrm{O}_{4}$ nanoparticles. These uniform and well-dispersed nanospheres were actually composed of loosely packed subunits with coarse surfaces, and disordered mesopores were distributed inside with high porosity. The hollow interior structure was further proved by the corresponding elemental line scan profile for iron and oxygen (Figure 1b). The marked interplanar spacings of 2.43, 2.51, and $2.95 \AA$ in the lattice fringe high-resolution TEM image
(Figure 1c) are consistent with the spacings of (222), (311), and (220) lattice planes $\left(d_{222}, d_{311}, d_{220}\right)$ for $\mathrm{Fe}_{3} \mathrm{O}_{4}$, respectively. The selected area electron diffraction (SAED) pattern (Figure 1d) taken from the nanospheres further exhibits the typical spotty diffraction rings of the polycrystalline structure, which could be well indexed to the lattice planes of the cubic spinel $\mathrm{Fe}_{3} \mathrm{O}_{4}$ phase. Furthermore, the Fe 2p photoemission spectrum of the sample (Figure 1e) showed no observable satellite structure, and two dominant peaks located at 710.6 and $724.2 \mathrm{eV}$ assignable to the Fe $2 \mathrm{p}_{3 / 2}$ and $\mathrm{Fe} 2 \mathrm{p}_{1 / 2}$ spin-orbit components, which is typical for the $\mathrm{Fe}_{3} \mathrm{O}_{4}$ spectrum. Thermogravimetric analysis (TGA) for magnetite $\mathrm{Fe}_{3} \mathrm{O}_{4}$ (Figure 1f) was performed to estimate the content of polymer groups or organic species bound on the surface. Based on the TGA curve and the corresponding derivative peaks, three-stage weight loss was observed, which might be related to the surface desorption of guest water molecules prior to $150{ }^{\circ} \mathrm{C}$, the drastic thermal degradation of polymer layers thereafter, and the further incineration of the residues, respectively. While considering that $\mathrm{Fe}_{3} \mathrm{O}_{4}$ conversion into $\mathrm{Fe}_{2} \mathrm{O}_{3}$ in air would give rise to a mass increase ${ }^{[16]}$ (starting from $\approx 150{ }^{\circ} \mathrm{C}$ ), the calculated weight fraction of organic ligands or layers should then be $\approx 8.3 \%$, instead of the experimentally observed $\approx 5.0 \%$ weight loss.

Figure 2a,b displays the representative SEM and TEM images for Mag-FeMn. As generally observed, the magnetite core exhibited considerable stability and retained an intact structure and morphology during the synthesis process. The successful deposition and encapsulation procedure was verified from the presence of numerous tiny aggregates covering the surface of Mag-Mn (Figure S2a, Supporting Information) and the nanoplatelet coatings with varying thickness for Mag-Fe (Figure S2b, Supporting Information) and Mag-FeMn. Note that the Mag-FeMn surface consisted of rod-like structures as well, and some were even inset into the particles (Figure 2b), which might also be associated with vertical nanoplates. In addition,
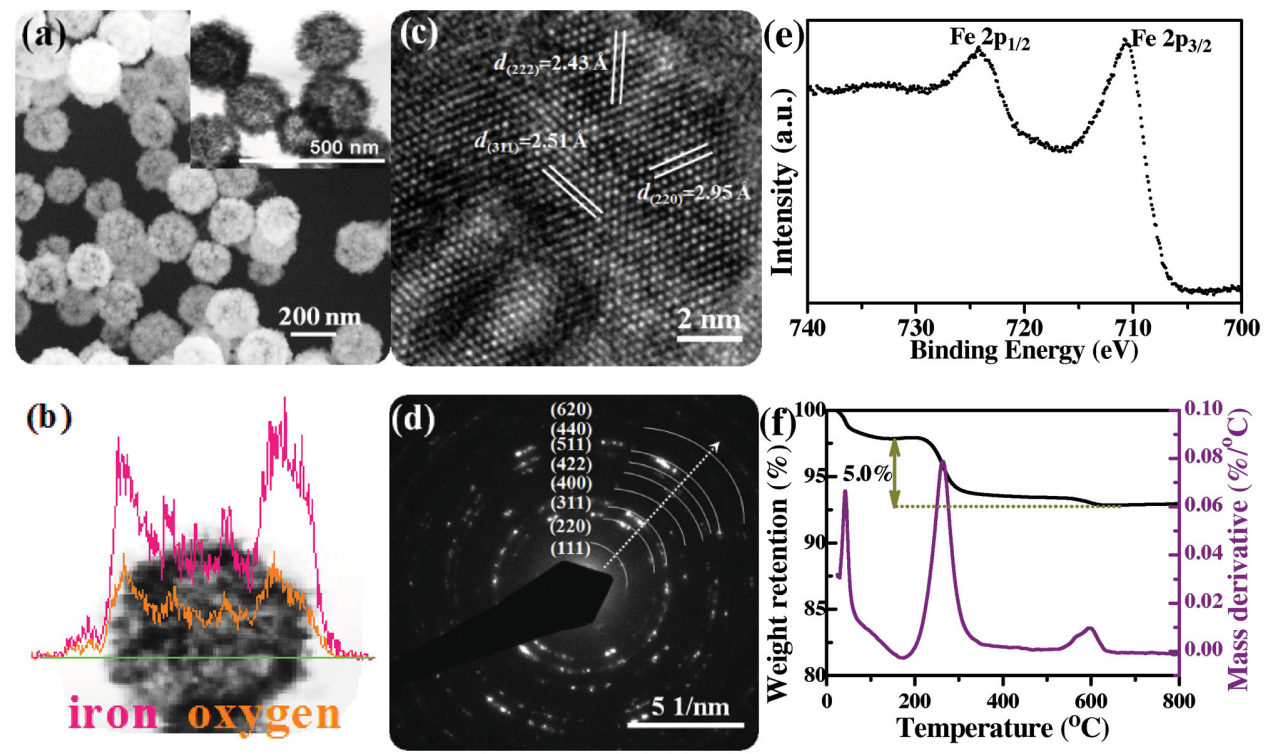

Figure 1. Characterization of the magnetic core. a) SEM and TEM (inset) micrographs, b) elemental line scan profile for as-synthesized $\mathrm{Fe}_{3} \mathrm{O}_{4}$ nanospheres. c) High magnification TEM images showing lattice planes indexed to $\mathrm{Fe}_{3} \mathrm{O}_{4}$. d) SAED pattern, e) XPS Fe 2p spectrum, and f) TGA analysis of $\mathrm{Fe}_{3} \mathrm{O}_{4}$. 

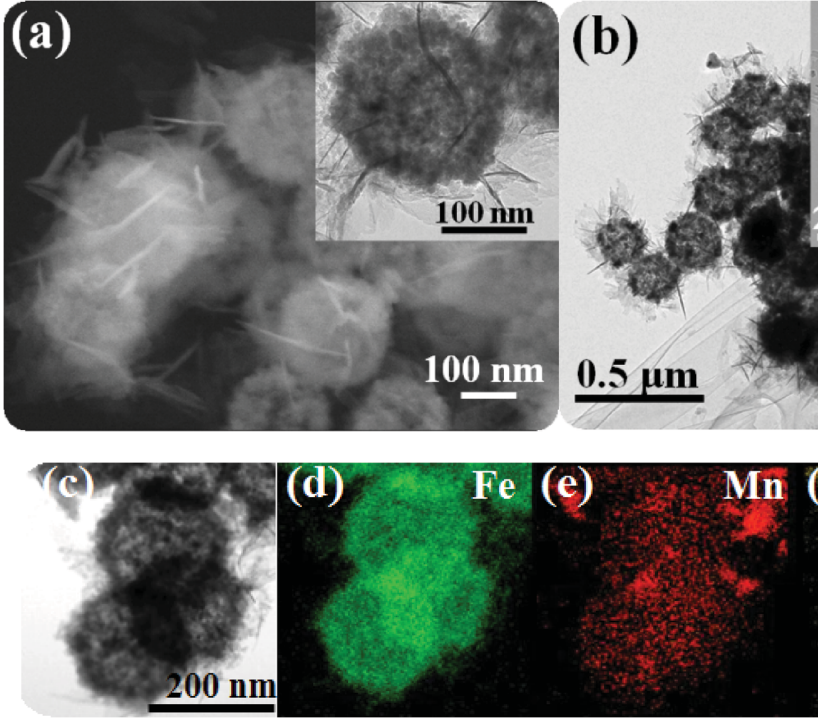

Figure 2. a,b) SEM and TEM micrographs for Mag-FeMn, and c-f) typical EDX elemental mappings of as-synthesized Mag-FeMn.

the energy-dispersive X-ray spectroscopy (EDX) element mappings of Mag-FeMn (Figure 2c-f) clearly confirmed the uniform distribution of $\mathrm{Fe}, \mathrm{Mn}$, and $\mathrm{O}$ elements in the heterostructure.

X-ray diffraction (XRD) diffractograms of the as-synthesized products are shown in Figure 3a. The characteristic peaks at $2 \theta$ of $18.3^{\circ}, 30.0^{\circ}, 35.4^{\circ}, 43.0^{\circ}, 53.4^{\circ}, 57.0^{\circ}, 62.5^{\circ}$, and $73.9^{\circ}$ can be readily indexed to (111), (220), (311), (400), (422), (511), (440), and (533) reflections of face-centered cubic $\mathrm{Fe}_{3} \mathrm{O}_{4}$ crystal (space group: Fd-3m, JCPDS 19-0629). Rietveld refined XRD patterns
The absorption bands at 2920, 2850, and $1454 \mathrm{~cm}^{-1}$ are associated with the stretching and bending vibrations of $\mathrm{C}-\mathrm{H}$ bonds (specifically, $v_{\mathrm{as}}(\mathrm{C}-\mathrm{H}), v_{\mathrm{s}}(\mathrm{C}-\mathrm{H})$, and $\delta(\mathrm{C}-\mathrm{H}))$, respectively, while the characteristic peak appeared near $1320 \mathrm{~cm}^{-1}$ should correspond to the $\mathrm{O}-\mathrm{H}$ bending vibration band. ${ }^{[18]}$ The FTIR results clearly confirmed that the $\mathrm{Fe}_{3} \mathrm{O}_{4}$ nanosphere was decorated with abundant carboxyl groups and hydroxyl groups, which should be beneficial for further functionalization on the surface via ligand coupling interactions. Surface modification with anchoring of functional groups was
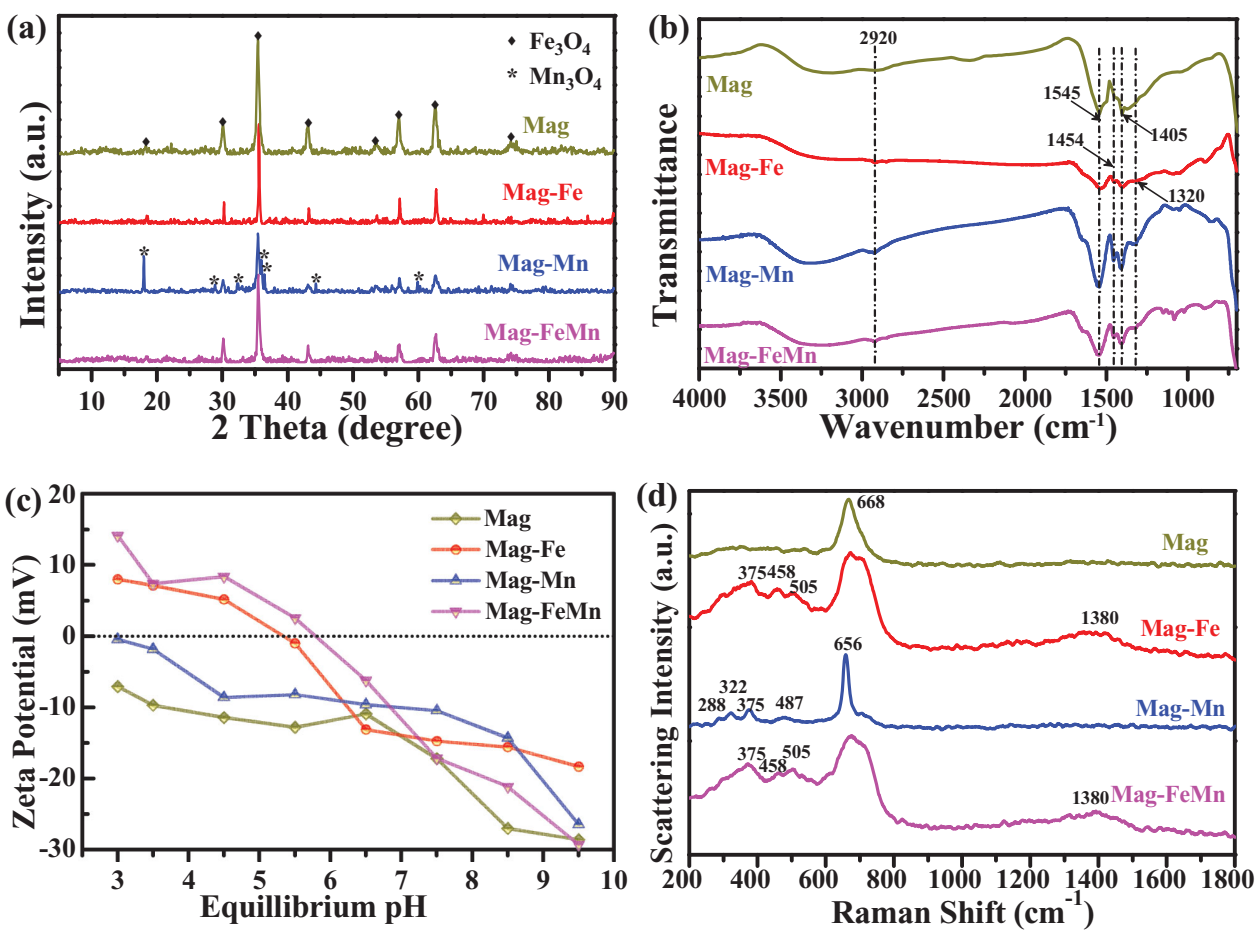

Figure 3. a) XRD patterns, b) FTIR spectra, c) Zeta potentials at different $\mathrm{pH}$, and d) Raman spectra for the prepared magnetic composites. 


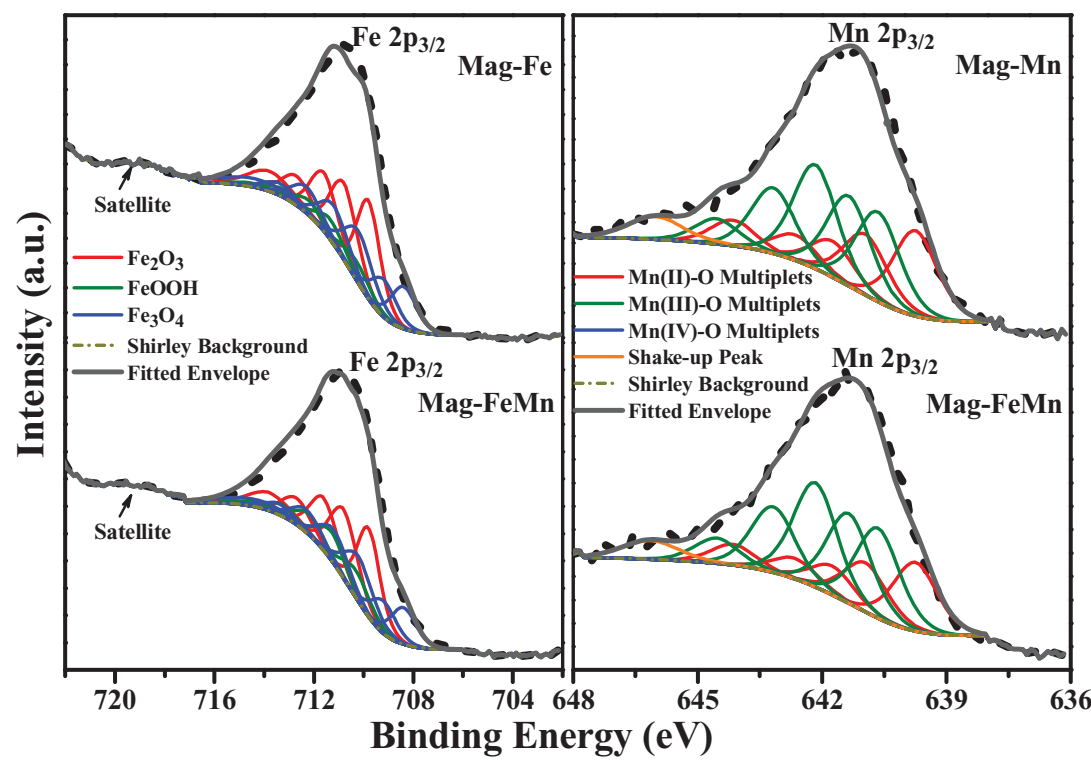

Figure 4. XPS Fe $2 p_{3 / 2}$ (left) and $M n 2 p_{3 / 2}$ (right) spectral interpretation and resolution of multiplet structures. For each chemical state, only the lowest-binding-energy peak intensity was allowed to adjust independently, while other peak intensities were calculated relative to it with fixed ratios. Spectral fitting was implemented by constructing the parameter-constrained GL(50) (Gaussian: Lorentzian 50:50) peaks for multiplet structure.

also reflected by the variation of zeta potential in the investigated $\mathrm{pH}$ range (Figure $3 \mathrm{c}$ ), which is directly related to the properties of charged groups on the surface. Overall, the Zeta potential measurements revealed that the amount of negative charges increased with increasing $\mathrm{pH}$, although with significant differences in potential values among the adsorbents. Note that the Mag displayed negative zeta potentials even at low $\mathrm{pH}$ value, possibly ascribed to the influence of the surface-bound carboxylic groups. ${ }^{[19]}$ This also verified the efficient carboxylic functionalization on the Mag surface. It should be noted as well that Mag-Fe, Mag-Mn, and Mag-FeMn showed higher zeta potentials relative to Mag, as an indication of the coordination of carboxylic groups with metal precursors in the post-encapsulation process, which weakened the contribution of $-\mathrm{COO}^{-}$ groups to the negative charges and zeta potential values.

The chemical compositions and structural properties of the composites were further investigated with Raman spectroscopy, which has been confirmed to be a powerful and complementary tool for resolving chemical states and molecular-specific structure for both crystalline and amorphous materials. ${ }^{[20]}$ The intense phonon band at $668 \mathrm{~cm}^{-1}$ observed for Mag was considered to be characteristic feature of magnetite $\mathrm{Fe}_{3} \mathrm{O}_{4}$, which should be assigned to the $\mathrm{A}_{1 \mathrm{~g}}$ Raman mode. ${ }^{[21]}$ Additional expected Raman optical phonons corresponding to the peaks at $300\left(E_{\mathrm{g}}\right)$ and $532 \mathrm{~cm}^{-1}\left(T_{2 \mathrm{~g}}\right)$ in the magnetite spectrum, which have also been reported, ${ }^{[12]}$ however, were not clearly detectable here, likely due to their weak intensity. The Raman spectrum of Mag-Mn recorded in Figure 3d displays Raman peaks near 288, 322, and $487 \mathrm{~cm}^{-1}$ accompanied by an intense narrow band at $656 \mathrm{~cm}^{-1}$. This indicated the presence of the $\mathrm{Mn}_{3} \mathrm{O}_{4}$ component, in agreement with reported data. ${ }^{[22]}$ The weak phonon band at $375 \mathrm{~cm}^{-1}$ could possibly be attributed to a minor amount of $\mathrm{Fe}_{3} \mathrm{O}_{4}$ conversion to $\gamma-\mathrm{Fe}_{2} \mathrm{O}_{3}$. As for Mag-Fe and Mag-FeMn, there was very little difference in the determined Raman modes, band positions, and assignments. No characteristic features of manganese oxides were discernible in the Raman spectrum of Mag-FeMn, due to overlapping of several frequencies in this band region. Taking account of the results of $\mathrm{XRD}$, we deduced that the appearance of the $1380 \mathrm{~cm}^{-1}$ band accompanied by other peaks at positions around 375,458 , and $505 \mathrm{~cm}^{-1}$ might originate from the formation of $\mathrm{Fe}_{2} \mathrm{O}_{3}$, with probably amorphous ferric oxyhydroxide. The broad band situated at around $650-720 \mathrm{~cm}^{-1}$ with a variable asymmetric shape suggested that a mixture of compounds coexisted. Due to the sample heterogeneity, the closely spaced frequencies, and the difficulty in establishing the exact band positions for different iron oxides and oxyhydroxides from the literature, ${ }^{[20 \mathrm{a}]}$ certain challenges were present in attempting to clearly resolve the chemical composition. In our case, spectrum interpretation was also based on the experimental conditions and XRD data, and the iron species were assigned to be mainly $\mathrm{Fe}_{3} \mathrm{O}_{4}$ with the oxidative product $\gamma-\mathrm{Fe}_{2} \mathrm{O}_{3}$, and amorphous $\mathrm{FeOOH}$ or $\mathrm{Fe}_{2} \mathrm{O}_{3}$.

Fe $2 p_{3 / 2}$ and $\mathrm{Mn} 2 \mathrm{p}_{3 / 2}$ X-ray photoelectron spectra (XPS) analyses of the prepared adsorbents were performed as well for surface chemical state identification (Figure 4). It should be noted that both iron and manganese include various oxidation states, among which $\mathrm{Mn}(\mathrm{II}), \mathrm{Mn}(\mathrm{III}), \mathrm{Mn}(\mathrm{IV}), \mathrm{Mn}(\mathrm{VI})$, and high-spin $\mathrm{Fe}(\mathrm{II}), \mathrm{Fe}(\mathrm{III})$ species contain unpaired $d$ electrons and give rise to complex multiplet splitting. ${ }^{[23]}$ In addition, the peak asymmetries and spectral overlaps in identification of mixed species further complicate interpretation of XPS spectra for both qualitative assignment and quantitative estimate of the different species. ${ }^{[23 c]}$ Such challenges were partially surmounted by earlier extensive attempts on resolution of multiplet structure toward consistently credible and reproducible spectral analysis. ${ }^{[23,24]}$ The fitting parameters and spectral features could then be used as guides for us to initially resolve XPS spectra and to further estimate the surface chemical information. Based on the well-resolved spectra achieved in previous reports, we attempted to employ a series of multiplet peaks for curve fitting of the multiple chemical states, and the derived peak parameters including binding energy, full width at half-maximum, and peak intensity are summarized in Table S1 (Supporting Information). With regard to interpretation of $\mathrm{Fe} 2 \mathrm{p}_{3 / 2}$ spectra, a quantitative estimate of the compositions was obtained under the assumption of that only $\mathrm{Fe}_{2} \mathrm{O}_{3}, \mathrm{FeOOH}$, and $\mathrm{Fe}_{3} \mathrm{O}_{4}$ phases might reside on the surface, and any satellites were ignored for simplification. However, for Mn $2 \mathrm{p}_{3 / 2}$ spectra, a relatively broad shake-up charge transfer satellite located at higher binding energy was generally observed, which is typically considered for $\mathrm{Mn}(\mathrm{II})$-containing compositions. ${ }^{[25]}$ The results of XPS analysis (Table S2, Supporting Information) consequently gave the nearsurface compositions of $\mathrm{Fe}_{2} \mathrm{O}_{3}: \mathrm{FeOOH}: \mathrm{Fe}_{3} \mathrm{O}_{4}$ in the proportions 47.9:12.0:40.1 for Mag-Fe, 45.4:18.0:36.6 for Mag-FeMn, 
and $\mathrm{Mn}(\mathrm{II}): \mathrm{Mn}(\mathrm{III}): \mathrm{Mn}(\mathrm{IV})$ in the percent of 38.1:61.1:0.8 for Mag-Mn, 33.2:66.3:0.5 for Mag-FeMn, with a close approximation of the composition ratio for $\mathrm{Mn}_{3} \mathrm{O}_{4}$.

As expected, $\mathrm{N}_{2}$-sorption measurements and the derived pore size distribution analyses (Figure S3, Supporting Information) further testified to the highly porous structure of the prepared adsorbents. The results on specific surface area $\left(S_{\mathrm{BET}}\right)$, pore diameter $\left(D_{\mathrm{P}}\right)$, and pore volume $\left(V_{\mathrm{P}}\right)$ are gathered in Table S3 (Supporting Information). The isotherms clearly delineated the characteristics of type IV curves according to the IUPAC classification, indicating pronounced mesoporosity or a certain degree of macroporosity for all samples, which was further demonstrated from the corresponding pore size distribution plots. In addition, the large hysteresis loops exhibited of type $\mathrm{H} 3$, while traditionally associated with the characteristics of capillary condensation and delayed evaporation processes in slit-shaped pores, were also found in isotherms of particles with mesoporous matrixes or hollow structures assembled with mesoporous walls. ${ }^{[26]}$ It was reasonable to conclude, then, the obtained wide hysteresis loops were due to the large amounts of interparticle voids present, which could also be observed from TEM micrographs. The very weak sorption at relative low pressure implied the absence of micropores, while the steep shape of adsorption branches $\left(P / P_{0}>0.85\right)$ was indicative of a narrow mesopore size distribution and an open-pore system inside the samples. This endowed the Mag with higher $S_{\mathrm{BET}}\left(48.3 \mathrm{~m}^{2} \mathrm{~g}^{-1}\right)$ and $V_{\mathrm{P}}\left(0.144 \mathrm{~cm}^{3} \mathrm{~g}^{-1}\right)$, with mesopore size centered at $23.3 \mathrm{~nm}$, compared to that of other reported hollow $\mathrm{Fe}_{3} \mathrm{O}_{4}$ microstructures. ${ }^{[27]}$ However, for the encapsulated magnetic nanoparticles (Mag-Fe, Mag-Mn, Mag-FeMn), although the porous structure was retained, reduced $S_{\mathrm{BET}}, V_{\mathrm{p}}$, and $D_{\mathrm{p}}$ were generally observed, likely due to the pore-blocking effect resulting from the growth of metal oxides on the outer shell.

The temperature dependence of the magnetic properties of $\mathrm{Fe}_{3} \mathrm{O}_{4}$ core was investigated after zero-field cooling (ZFC) and field cooling (FC, H = $100 \mathrm{Oe}$ ). Magnetic moment measurements were conducted with increasing temperature up to $300 \mathrm{~K}$ with a 100 Oe probe field. For the ZFC process at a very low temperature, the net magnetic moment approaches nearly zero due to the magnetization aligning along its easy axis with random orientation, and high energy barrier arising from magnetic anisotropy in the so-called blocked state. As temperature rises, more and more nanoparticles, especially those with low energy barriers, can be able to have their energy barriers surmounted by the increasing thermal activation energy. ZFC magnetization thus increases, since magnetic moments start to be switched away from the easy axis and progressively reorient parallel to the applied field. Note that in the blocked state, the relaxation time of the magnetic moment is short compared to the experimental characteristic times. ${ }^{[28]}$ The magnetic system cannot reach thermodynamic equilibrium, and magnetization irreversibility results in the departure between ZFC and FC magnetization. The magnetization approaches a maximum in a transition point referred to as the blocking temperature $\left(T_{\mathrm{B}}\right)$, at which the thermal activation energy becomes balanced with the energy barrier of magnetic anisotropy. The nanoparticles exhibit superparamagnetic properties at $T_{\mathrm{B}}$, beyond which magnetic moments experience thermal fluctuations and flip rapidly within experimental time scales, accompanied by a magnetization decrease. Since nanoparticles are at the same superparamagnetic relaxation state, magnetic moment evolution coincides above $T_{\mathrm{B}}$ for the $\mathrm{FC}$ and $\mathrm{ZFC}$ processes. In the present study, complete separation for the FC and ZFC curves was observed with $T_{\mathrm{B}}>300 \mathrm{~K}$ under the investigated temperature range (Figure 5a), implying the presence of characteristic ferromagnetic behavior. ${ }^{[28 d]}$ In addition, the flat-shaped FC curve, especially at low temperature, suggested strong interparticle interactions in stabilizing magnetic moments or the development of a collective magnetic state. ${ }^{[29]}$ As expected, field-dependent magnetization behavior of the prepared magnetic nanoparticles was also observed, as reflected in Figure 5b with obvious hysteresis loops, which are typical for ferromagnetic-type curves. The saturation magnetization $\left(M_{\mathrm{s}}\right)$ for Mag was estimated to be $72.3 \mathrm{emu} \mathrm{g}^{-1}$, and relatively low saturation magnetization values were reasonably obtained for Mag-Fe (20.2 emu g $\left.{ }^{-1}\right)$, Mag-Mn (50.0 emu g ${ }^{-1}$ ), and Mag-FeMn (31.0 emu g $\left.{ }^{-1}\right)$ as a result of the nonmagnetic coatings on the surface. The particles exhibited good magnetic response, and thus effective magnetically driven recovery could be anticipated. Specifically, as reflected in Figure S4 (Supporting Information), Mag-FeMn composites showed satisfactory magnetic-responsive retrieval and unaffected redispersion behavior in aqueous solution simply by applying or removing an external magnetic field.

The mesoporous Mag-FeMn can be considered a potential candidate for aqueous As(III) removal, as was evidenced from the corresponding adsorption isotherms in comparison with other adsorbents (Figure 6a). Langmuir and Freundlich models were employed for isotherm fitting, and the detailed fitting parameters are summarized in Table S4 (Supporting Information). Both adsorption models well described As(III) uptake behavior on the prepared sorbents, except for Mag, based on the derived correlation coefficient $\left(r^{2}\right)$. Note that Mag-FeMn exhibited a quite competitive high capacity for As(III) capture (56.1 $\mathrm{mg} \mathrm{g}^{-1}$ ), higher than that of the Mag, Mag-Mn, and Mag-Fe (from 5.0 to $40.7 \mathrm{mg} \mathrm{g}^{-1}$ ), in terms of the Langmuir adsorption model employed. Similar results could be obtained as well by comparison of the Freundlich constants $\left(K_{\mathrm{F}}\right)$, which represent the adsorption affinity derived from the Freundlich model. In principle, high surface areas and pore structures usually imply 

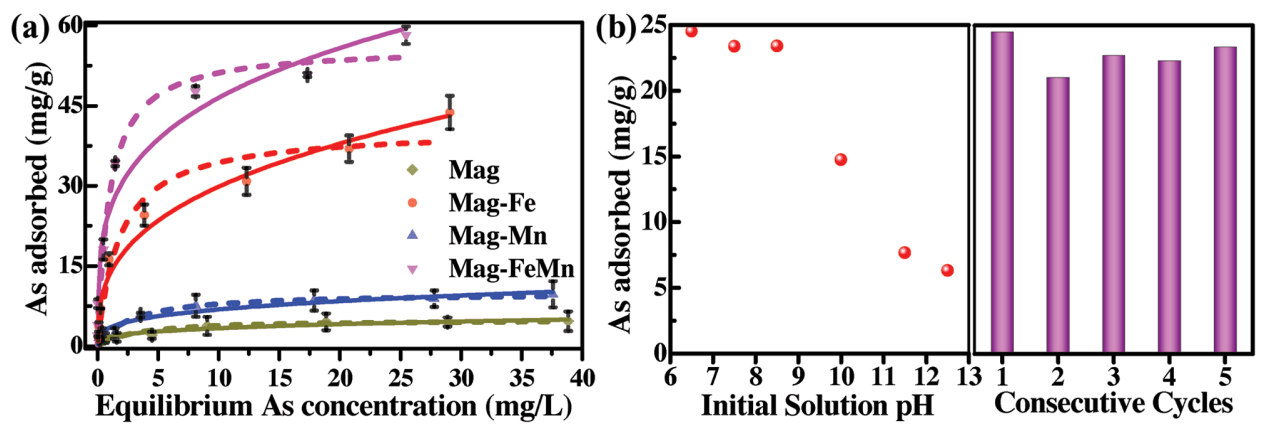

Figure 6. a) Adsorption isotherms of $\mathrm{As}$ (III) at pH 6.5 in $0.01 \mathrm{M} \mathrm{NaNO}_{3}$ as background electrolytes. The solid lines and dashed lines represent Freundlich and Langmuir isotherm fittings, respectively. b) Effect of initial solution $\mathrm{pH}$ on As(III) sorption over Mag-FeMn composite and its adsorption capacities for As (III) in five consecutive cycles (initial As(III) concentration $0.1 \times 10^{-3} \mathrm{M}$, regenerant $0.2 \mathrm{M} \mathrm{NaOH}$ ).

enhanced mass-transfer and accessibility of binding sites, while the as-synthesized Mag showed an extremely poor sorption capacity for As(III). Further, similar surface areas and pore sizes also gave rise to the completely different sorption capacities for Mag-Fe, Mag-Mn, and Mag-FeMn. It should be then emphasized that the performances of these four adsorbents were not dictated by surface areas and pore structures. Actually, the efficient $\mathrm{As}(\mathrm{III})$ removal has always been particularly problematic in water pollution control and quality restoration, due to the predominant uncharged species $\left(\mathrm{H}_{3} \mathrm{AsO}_{3}\right)$ of arsenite at neutral $\mathrm{pH}$, and the relatively weak affinity to adsorbents. ${ }^{[30]}$ Hence, even though iron oxide-based materials usually exhibit improved efficiencies for arsenic removal, a preoxidation process for As(III) prior to sorption is necessary, due to the more superior affinity between iron oxides and As(V). In Mag-FeMnbased system, the coupled As(III) oxidation by manganese oxide with the synchronous As(V) sorption by $\mathrm{Fe}(\mathrm{III})$ oxide was considered to facilitate the removal of As(III). The hierarchically structured Mag-FeMn with nanoplatelet-like shell would also be supposed to possess of promoted mass-transfer ability for chemical/physical processes.

Note also that $\mathrm{As}(\mathrm{III})$ sorption on Mag-FeMn was a $\mathrm{pH}$ dependent process, with rather weak sorption capacity at a higher $\mathrm{pH}$ (Figure 6b), suggesting the adsorbent could be regenerated in an extreme alkaline solution and harvested under an external magnetic field. As such, the regeneration of saturated Mag-FeMn was implemented simply by dispersion into $0.2 \mathrm{~m} \mathrm{NaOH}$ solution. No significant decrease of As(III) sorption capacity was observed throughout five consecutive cycles. Further examination of the TEM image for Mag-FeMn after sorption of As(III) showed no obvious change in morphology as well (Figure S5, Supporting Information). The recovered Mag-FeMn composites still retained nanoplatelet coatings with varying thickness and rod-like structures even inset into the nanospheres. Thus, limited component loss would also be anticipated, indicating the excellent stability of Mag-FeMn during sorption process. Considering the significantly higher adsorption affinity and saturation capacity of As(III) than other adsorbents in previously reported works (Table S5, Supporting Information), Mag-FeMn would be applied as a desirable and recoverable adsorbent in efficient arsenic removal.

With regard to kinetics, Mag-FeMn could almost completely remove aqueous $\mathrm{As}$ (III) $(1.0 \mathrm{ppm}$ ) within $60 \mathrm{~min}$, and a similar but slight compressed trend for As(III) sequestration was observed for Mag-Fe (Figure 7a). The fairly rapid initial depletion rate further confirmed facilitated diffusion and capture of As(III) on the sorption sites. By contrast, Mag and Mag-Mn exhibited little affinity for As(III), in line with the weak saturation capacity from the corresponding adsorption isotherm analysis above. As(III) and $\mathrm{As}(\mathrm{V})$ are the typical species for inorganic arsenic, and the transformation of As(III) to As(V) commonly takes place under oxidative conditions. In most cases, simple determination of $\mathrm{As}(\mathrm{III})$ is inadequate for fully understanding the likely mechanism, due to the dependence of both the mobility and sorption behavior of arsenic on its chemical speciation. As such, synchronous speciation analysis by HPLC-ICPMS was performed as well in quantifying the concentration changes of the aqueous transformed $\mathrm{As}(\mathrm{V})$ in the sorption process. Figure S6 (Supporting Information) displays the chromatographic separation of As species in standard solutions with different concentration ranges. Note that a relatively higher amount of residual $\mathrm{As}(\mathrm{V})$ was observed, and up to $\approx 50 \mu \mathrm{g} \mathrm{L}^{-1}$ of $\mathrm{As}(\mathrm{V})$ in the initial period was found for the Mag-Mn based system, compared with the other adsorbents (Figure 7b). With its high reducing potential, $\mathrm{Mn}_{3} \mathrm{O}_{4}$ has already been reported to show great promise in heterogeneous catalysis, such as in
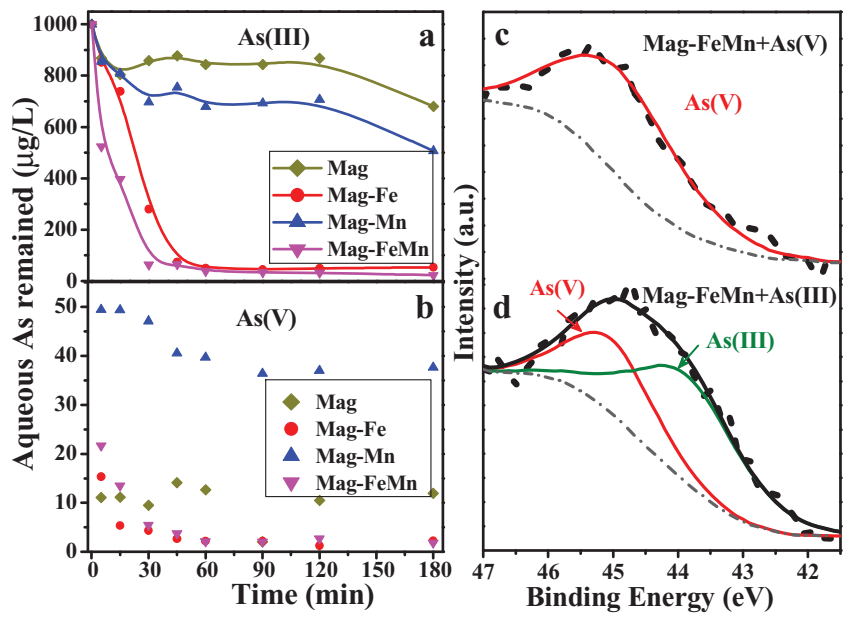

Figure 7. a,b) Temporal variation of aqueous concentrations of As(III) and $\mathrm{As}(\mathrm{V})$ in Mag-FeMn based system. Initial As(III) concentration $1.0 \mathrm{mg} \mathrm{L}^{-1}$, adsorbent dose $0.25 \mathrm{~g} \mathrm{~L}^{-1}$. c,d) XPS As 3d spectra of Mag$\mathrm{FeMn}$ after reaction with $\mathrm{As}(\mathrm{V})$ and $\mathrm{As}(\mathrm{III})$ with initial concentration of $20 \mathrm{mg} \mathrm{L}^{-1}$, respectively. 
dye decolorization and oxidation of methane and carbon monoxide, and polymerization of aniline monomer. ${ }^{[31]}$ It was then reasonable to expect that the generation of $\mathrm{As}(\mathrm{V})$ was derived from the oxidation of $\mathrm{As}$ (III) by $\mathrm{Mn}_{3} \mathrm{O}_{4}$. Note also that a relatively low concentration of aqueous $\mathrm{As}(\mathrm{V})$ was detected in the sorption process using Mag-FeMn, which was due to the simultaneous sorption of the $\mathrm{As}(\mathrm{V})$ newly generated from $\mathrm{As}(\mathrm{III})$ oxidation. The co-occurring redox reaction involved was also partly verified from the slight increase of content of surface $\mathrm{Mn}(\mathrm{II})$ in comparison with the pristine Mag-FeMn after sorption of As(III) (Table S2 and Figure S7, Supporting Information). However, the predominantly surface Mn(III) (59.6\%) still exhibited great potential to oxidize As(III), leading to satisfactory sorption capacities during consecutive cycles (Figure 6b). XPS was further employed to examine the evolution of arsenic oxidation states after adsorption equilibrium had been reached on the surface of Mag-FeMn. Comparison of the As 3d spectra after individual interaction with $\mathrm{As}(\mathrm{V})$ (Figure 7c) and $\mathrm{As}(\mathrm{III})$ (Figure 7d) proved that synchronous oxidation was involved in As(III) sorption. Two fitted peaks located near 44.1 and $45.2 \mathrm{eV}$ indeed revealed the presence of $\mathrm{As}(\mathrm{III})$ and $\mathrm{As}(\mathrm{V})$ on the surface of Mag-FeMn for As(III) sorption. A likely As(III) removal mechanism was hereby proposed involving simultaneous oxidation and adsorption in the Mag-FeMn based system.

\section{Conclusions}

Magnetically-confined Fe-Mn bimetallic oxide encapsulation was controllably synthesized by cooperative assembly of $\mathrm{Fe}_{3} \mathrm{O}_{4}$ and FMBO. The synthesis procedure could endow the magnetic core with abundant carboxyl groups conjugated on the surface, and thus superior dispersibility, hydrophilicity, and wettability for direct surface encapsulation in virtue of the expected low interfacial energy. The controllable postdeposition process further ensured the uniform distribution of iron and manganese oxides. The derived composites retained a highly porous structure with good magnetic response. The likely mechanism for the enhanced removal of As(III) was further deduced, with simultaneous oxidation and adsorption processes involved. Considering the excellent adsorption capacity and convenient recoverability associated with the facile magnetic separation, Mag-FeMn would show particular promise for development of nanotechnology enabled approaches for adsorption-based water purification.

\section{Experimental Section}

Chemicals and Reagents: Iron (III) chloride hexahydrate $\left(\mathrm{FeCl}_{3} \cdot 6 \mathrm{H}_{2} \mathrm{O}\right)$, HMTA, manganese(II) acetate tetrahydrate $\mathrm{Mn}\left(\mathrm{CH}_{3} \mathrm{COO}\right)_{2} \bullet 4 \mathrm{H}_{2} \mathrm{O}$, trisodium citrate dihydrate $\left(\mathrm{Na}_{3} \mathrm{C}_{6} \mathrm{H}_{5} \mathrm{O}_{7} \cdot 2 \mathrm{H}_{2} \mathrm{O}\right)$, urea, PAM (MW 3 000000 ), and PVP (grade K30, MW 40000 ) were all at least of analytical grade from Sinopharm Chemical Reagent Co., Ltd. $\mathrm{NaAsO}_{2}$ and $\mathrm{Na}_{3} \mathrm{AsO}_{4} \cdot 12 \mathrm{H}_{2} \mathrm{O}$ were used for preparation of the stock solutions of As (III) and As(V).

Materials Synthesis: The porous $\mathrm{Fe}_{3} \mathrm{O}_{4}$ nanospheres were obtained via a modified hydrothermal reaction. ${ }^{[32]}$ Briefly, $\mathrm{FeCl}_{3} \cdot 6 \mathrm{H}_{2} \mathrm{O}(1.08 \mathrm{~g})$, $\mathrm{Na}_{3} \mathrm{C}_{6} \mathrm{H}_{5} \mathrm{O}_{7} \cdot 2 \mathrm{H}_{2} \mathrm{O}(2.35 \mathrm{~g})$, and urea $(0.9 \mathrm{~g})$ together with PAM $(0.6 \mathrm{~g})$ were dissolved in an $80 \mathrm{~mL}$ aqueous solution under vigorous magnetic stirring to form a transparent solution. After addition of $0.3 \mathrm{~g}$ PVP, the resulting homogeneous solution was transferred to a Teflonlined autoclave and kept at $200{ }^{\circ} \mathrm{C}$ for $18 \mathrm{~h}$. The yielded precipitate was harvested and ultrasonically washed with water and ethanol repeatedly before drying at $60^{\circ} \mathrm{C}$ under vacuum. The obtained magnetic nanospheres were used as seeds for the subsequent synthesis of Mag$\mathrm{Fe}$, Mag-Mn (MNPs enwrapped solely with either iron or manganese oxide), and Mag-FeMn.

In a typical procedure, $\mathrm{Fe}_{3} \mathrm{O}_{4}$ nanoparticles were first ultrasonically dispersed in aqueous PVP solution, followed by the addition of HMTA, and iron and/or manganese precursors. The final reaction mixture $(40 \mathrm{~mL})$, containing $110 \times 10^{-3} \mathrm{M}$ PVP (monomer concentration),

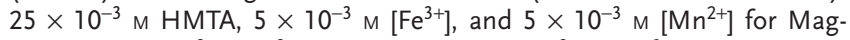
FeMn $\left(10 \times 10^{-3} \mathrm{M}\left[\mathrm{Fe}^{3+}\right]\right.$ for Mag-Fe, $10 \times 10^{-3} \mathrm{M}\left[\mathrm{Mn}^{2+}\right]$ for Mag-Mn), was incubated at $95^{\circ} \mathrm{C}$ for $5 \mathrm{~h}$. The products were magnetically collected and washed with water and ethanol before drying at $80^{\circ} \mathrm{C}$ overnight.

As (III) Adsorption Test: The adsorption kinetic studies were conducted by dispersion of the corresponding sorbent (100 mg Fe${ }_{3} \mathrm{O}_{4}$, Mag$\mathrm{Fe}, \mathrm{Mag}-\mathrm{Mn}$, or Mag-FeMn) into $400 \mathrm{~mL} \mathrm{As}$ (III) solution with initial concentration of $1.0 \mathrm{mg} \mathrm{L}^{-1}$. The initial $\mathrm{pH}$ of the sample was adjusted to the value of $6.5 \pm 0.1$ with $0.1 \mathrm{M} \mathrm{HNO}_{3}$, and a constant ionic strength of $0.01 \mathrm{M}$ was maintained with $\mathrm{NaNO}_{3}$. A designated amount of suspension $(1.0 \mathrm{~mL})$ was withdrawn at given intervals, and passed through a $0.22 \mu \mathrm{m}$ membrane filter. The collected filtrate was subjected to speciation analysis of $A s(I I I)$ and $A s(V)$, which was achieved by high performance liquid chromatography (HPLC, Agilent 1200) separation using a Hamilton PRP-X100 HPLC column followed by determination with inductively coupled plasma mass spectrometry (ICP-MS, Agilent 7500a). The isocratic mobile phase consisted of $15 \times 10^{-3} \mathrm{M}\left(\mathrm{NH}_{4}\right)_{2} \mathrm{HPO}_{4}$

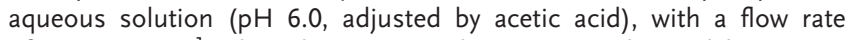
of $1.2 \mathrm{~mL} \mathrm{~min}$. The adsorption isotherms were obtained by varying the initial $\mathrm{As}$ (III) concentration from 0.5 to $40 \mathrm{mg} \mathrm{L}^{-1}$. After sorption equilibrium for $24 \mathrm{~h}$, the suspension was magnetically separated and the remaining arsenic in solution was measured by inductively coupled plasma-optical emission spectroscopy (Optima 8300, PerkinElmer). The regeneration of saturated Mag-FeMn was implemented simply by dispersion into $0.2 \mathrm{~m} \mathrm{NaOH}$ solution and recovered through magnetic separation as well.

Materials Characterization: The morphologies and microstructures of the samples were examined on a field emission SEM (ZEISS Merlin Compact) and TEM (JEOL JEM-2010F; H-7500, Hitachi). The elemental mapping images were recorded using EDX (Oxford Instruments). The adsorbent constituents, crystal structures, and surface properties were characterized by Powder XRD (Panalytical X'Pert Pro MPD diffractometer), FTIR (Nicolet 5700), confocal Raman microspectroscopy (inVia-Reflex, Renishaw), and X-ray photoelectron spectroscopy (AXIS Ultra DLD, Kratos Analytical) with a monochromatic Al $\mathrm{K} \alpha$ source $(1486.7 \mathrm{eV})$. Raman scattering was excited under $632.8 \mathrm{~nm}$ radiation, with laser power at the sample always kept below $0.1 \mathrm{~mW}$ to minimize laser-induced conversion of samples. For XPS analyses, all of the measured binding energies were charge-calibrated to the $\mathrm{C} 1 \mathrm{~s}$ peak of adventitious carbon ( $\mathrm{C}-\mathrm{C}, \mathrm{C}-\mathrm{H}$ ) set to $284.8 \mathrm{eV}$. Nitrogen adsorptiondesorption isotherms were obtained at $77 \mathrm{~K}$ on a gas adsorption analyzer instrument (ASAP 2020 HD88, Micromeritics). A Netzsch STA449 F3 thermal analyzer was employed for TGA from ambient temperature to $800{ }^{\circ} \mathrm{C}$ under air flow, with a heating rate of $10{ }^{\circ} \mathrm{C} \mathrm{min}-1$. Zeta potentials were determined on a particle size and Zeta-potential analyzer (DelsaNano C, Beckman Coulter). Magnetic properties were explored on a Physical Property Measurement System (PPMS-9, Quantum Design). The temperature dependence of magnetization was investigated in zerofield-cooled (ZFC) and field-cooled (FC) conditions from 300 to $2 \mathrm{~K}$ with a 100 Oe probe field. The mass magnetizations were examined in an external field range from -20 to $20 \mathrm{kOe}$ at $300 \mathrm{~K}$.

\section{Supporting Information}

Supporting Information is available from the Wiley Online Library or from the author. 


\section{Acknowledgements}

This work was supported by the National Science Fund for Distinguished Young Scholars of China (Grant No. 51225805) and the Funds for Creative Research Groups of China (Grant No. 51221892).

Received: January 12, 2016 Revised: March 13, 2016 Published online: March 31, 2016

[1] a) Y. Zhang, M. Yang, X.-M. Dou, H. He, D.-S. Wang, Environ. Sci. Technol. 2005, 39, 7246; b) Z. Zhang, J. Liu, J. Gu, L. Su, L. Cheng, Energy Environ. Sci. 2014, 7, 2535; c) Y. Zhang, L. Li, H. Su, W. Huang, X. Dong, J. Mater. Chem. A 2015, 3, 43; d) M. A. Carreon, V. V. Guliants, Eur. J. Inorg. Chem. 2005, 2005, 27.

[2] a) W. Shi, H. Zeng, Y. Sahoo, T. Y. Ohulchanskyy, Y. Ding, Z. L. Wang, M. Swihart, P. N. Prasad, Nano Lett. 2006, 6, 875; b) R. Buonsanti, V. Grillo, E. Carlino, C. Giannini, F. Gozzo, M. Garcia-Hernandez, M. A. Garcia, R. Cingolani, P. D. Cozzoli, J. Am. Chem. Soc. 2010, 132, 2437.

[3] G. Zhang, F. Liu, H. Liu, J. Qu, R. Liu, Environ. Sci. Technol. 2014, 48, 10316.

[4] a) C. T. Yavuz, J. Mayo, W. Y. William, A. Prakash, J. C. Falkner, S. Yean, L. Cong, H. J. Shipley, A. Kan, M. Tomson, D. Natelson, V. L. Colvin, Science 2006, 314, 964; b) C. T. Yavuz, J. T. Mayo, C. Suchecki, J. Wang, A. Z. Ellsworth, H. D'Couto, E. Quevedo, A. Prakash, L. Gonzalez, C. Nguyen, C. Kelty, V. L. Colvin, Environ. Geochem. Health 2010, 32, 327.

[5] J.-P. Jolivet, C. Chaneac, E. Tronc, Chem. Commun. 2004, 481.

[6] a) S. Guo, S. Dong, E. Wang, Chem. Eur. J. 2009, 15, 2416; b) C. Wang, W. Tian, Y. Ding, Y.-q. Ma, Z. L. Wang, N. M. Markovic, V. R. Stamenkovic, H. Daimon, S. Sun, J. Am. Chem. Soc. 2010, 132, 6524; c) Y. Xia, X. Xia, H. C. Peng, J. Am. Chem. Soc. 2015, 137, 7947.

[7] a) J. Liu, S. Z. Qiao, Q. H. Hu, G. Q. Lu, Small 2011, 7, 425; b) L. Li, Y. Feng, Y. Li, W. Zhao, J. Shi, Angew. Chem. Int. Ed. 2009, 48, 5888; c) M. B. Gawande, P. S. Branco, R. S. Varma, Chem. Soc. Rev. 2013, 42, 3371; d) J. Tucek, K. C. Kemp, K. S. Kim, R. Zboril, ACS Nano 2014, 8, 7571; e) D. Wang, D. Astruc, Chem. Rev. 2014, 114, 6949.

[8] a) J. Zhang, Y. Tang, K. Lee, M. Ouyang, Science 2010, 327, 1634; b) Y. Feng, J. He, H. Wang, Y. Y. Tay, H. Sun, L. Zhu, H. Chen, J. Am. Chem. Soc. 2012, 134, 2004; c) H. Sun, J. He, J. Wang, S. Y. Zhang, C. Liu, T. Sritharan, S. Mhaisalkar, M. Y. Han, D. Wang, H. Chen, J. Am. Chem. Soc. 2013, 135, 9099.

[9] C. Shan, M. Tong, Water Res. 2013, 47, 3411.

[10] Z. Niu, Y. Li, Chem. Mater. 2014, 26, 72.

[11] a) X. W. Lou, L. A. Archer, Adv. Mater. 2008, 20, 1853; b) J. Ma, K. Wang, M. Zhan, ACS Appl. Mater. Interfaces 2015, 7, 16027.

[12] Z. Wang, L. Wu, M. Chen, S. Zhou, J. Am. Chem. Soc. 2009, 131, 11276.

[13] M. Feyen, C. Weidenthaler, F. Schüth, A.-H. Lu, J. Am. Chem. Soc. 2010, 132, 6791.
[14] Y. Zhao, L. Jiang, Adv. Mater. 2009, 21, 3621.

[15] a) Q. Peng, Y. Dong, Y. Li, Angew. Chem. Int. Ed. 2003, 115, 3135; b) X. Li, Y. Xiong, Z. Li, Y. Xie, Inorg. Chem. 2006, 45, 3493.

[16] H. Deng, X. Li, Q. Peng, X. Wang, J. Chen, Y. Li, Angew. Chem. Int. Ed. 2005, 117, 2842.

[17] S. Xuan, Y.-X. J. Wang, J. C. Yu, K. Cham-Fai Leung, Chem. Mater. 2009, 21, 5079.

[18] a) J. Zhang, R. Srivastava, R. Misra, Langmuir 2007, 23, 6342; b) V. Panwar, P. Kumar, A. Bansal, S. S. Ray, S. L. Jain, Appl. Catal. A: Gen. 2015, 498, 25.

[19] Y. Li, D. Yuan, M. Dong, Z. Chai, G. Fu, Langmuir 2013, 29, 11770.

[20] a) I. Chourpa, L. Douziech-Eyrolles, L. Ngaboni-Okassa, J.-F. Fouquenet, S. Cohen-Jonathan, M. Soucé, H. Marchais, P. Dubois, Analyst 2005, 130, 1395; b) J. Gao, M. A. Lowe, H. c. D. Abruña, Chem. Mater. 2011, 23, 3223.

[21] a) D. De Faria, S. Venâncio Silva, M. De Oliveira, J. Raman Spectrosc. 1997, 28, 873; b) O. N. Shebanova, P. Lazor, J. Solid State Chem. 2003, 174, 424.

[22] C.-C. Hu, Y.-T. Wu, K.-H. Chang, Chem. Mater. 2008, 20, 2890.

[23] a) H. Nesbitt, D. Banerjee, Am. Mineral. 1998, 83, 305; b) H. Nesbitt, G. Canning, G. Bancroft, Geochim. Cosmochim. Acta 1998, 62, 2097; c) M. C. Biesinger, B. P. Payne, A. P. Grosvenor, L. W. M. Lau, A. R. Gerson, R. S. C. Smart, Appl. Surf. Sci. 2011, 257, 2717.

[24] a) G. Lee, K. Song, J. Bae, Geochim. Cosmochim. Acta 2011, 75, 4713; b) T.-C. Lin, G. Seshadri, J. A. Kelber, Appl. Surf. Sci. 1997, 119, 83.

[25] I. V. Chernyshova, S. Ponnurangam, P. Somasundaran, J. Catal. 2011, 282, 25

[26] a) H.-P. Lin, S.-T. Wong, C.-Y. Mou, C.-Y. Tang, J. Phys. Chem. B 2000, 104, 8967; b) X.-F. Qu, Q.-Z. Yao, G.-T. Zhou, S.-Q. Fu, J.-L. Huang, J. Phys. Chem. C 2010, 114, 8734.

[27] Q. Q. Xiong, J. P. Tu, Y. Lu, J. Chen, Y. X. Yu, Y. Q. Qiao, X. L. Wang, C. D. Gu, J. Phys. Chem. C 2012, 116, 6495.

[28] a) A. J. Rondinone, A. C. Samia, Z. J. Zhang, J. Phys. Chem. B 1999 103, 6876; b) H. Cao, R. Liang, D. Qian, J. Shao, M. Qu, J. Phys. Chem. C 2011, 115, 24688; c) X. Sun, N. F. Huls, A. Sigdel, S. Sun, Nano Lett. 2012, 12, 246; d) N. Fontaína Troitiño, B. Rivas-Murias, B. Rodríguez-González, V. Salgueirino, Chem. Mater. 2014, 26, 5566.

[29] a) C. Cannas, A. Musinu, D. Peddis, G. Piccaluga, Chem. Mater. 2006, 18, 3835; b) J. Lu, X. Jiao, D. Chen, W. Li, J. Phys. Chem. C 2009, 113, 4012.

[30] a) V. K. Sharma, M. Sohn, Environ. Int. 2009, 35, 743; b) J. Li, Z. Shi, B. Ma, P. Zhang, X. Jiang, Z. Xiao, X. Guan, Environ. Sci. Technol. 2015, 49, 10581.

[31] a) A. N. Chowdhury, M. S. Azam, M. Aktaruzzaman, A. Rahim, J. Hazard. Mater. 2009, 172, 1229; b) E. Stobbe, B. De Boer, J. Geus, Catal. Today 1999, 47, 161; c) Z. Chen, Z. Jiao, D. Pan, Z. Li, M. Wu, C. H. Shek, C. M. Wu, J. K. Lai, Chem. Rev. 2012, 112, 3833.

[32] D. Chen, G. Ji, Y. Ma, J. Y. Lee, J. Lu, ACS Appl. Mater. Interfaces 2011, 3, 3078. 